\title{
Vida e historia en el pensamiento de Marx*
}

Fecha de entrega: 28 de octubre de 2018

Fecha de evaluación: 11 de diciembre de 2018

Fecha de aprobación: 30 de enero de 2019

\author{
Andrés Esteban Bejarano**
}

\section{Resumen}

La vida es el fundamento de los bienes, no un bien. El análisis de la sociedad moderna hace inevitable cuestionar: ¿Qué es la vida cuando se ha convertido en una mercancía? Es a través de ese concepto, vida, que se pueden advertir los movimientos de la historia. Su desarrollo genealógico en el decurso de los modos de producción es la condición de posibilidad para la comprensión del proceso histórico a través del materialismo dialéctico hasta llegar a la fase de desarrollo en la cual la vida entra en una profunda contradicción con su reproducción material. Entender cada momento histórico a la luz de las contradicciones entre las fuerzas productivas materiales, las relaciones de producción existentes y sus formas jurídicas ya no en relación con la materia, sino con la vida, es decir, con el sujeto, da un nuevo sentido a la comprensión de la historia, ya no como progreso, sino como proceso. Todo esto también conduce a cuestiones de carácter moral y al insondable

* Este texto es producto de la investigación y de los intereses desarrollados durante el pregrado en la Universidad La Gran Colombia, que serán profundizados en estudios de posgrado. Citar como: Bejarano, E. A. (2019). Vida e historia en el pensamiento de Marx. Cuadernos de Filosofía Latinoamericana, 40(121), 131-144. DOI: https://doi.org/10.15332/25005375.5474

* Estudiante de Licenciatura en Filosofía e Historia, Universidad La Gran Colombia. Correo electrónico: abejaranoi@ulagrancolombia.edu.co 
problema de las relaciones entre el modo de producción burgués, la vida, la apropiación de trabajo y la lógica del capital.

Palabras clave: materialismo, dialéctica, religión, capital y mercancía.

\section{Life and History in the Thought of Marx}

\section{Abstract}

Life is not a good, but rather, the ground of all goods. When analyzing modern society it is inevitable to ask ourselves: What is life when it has become a commodity? The concept of life makes it possible to grasp the movements of history. Its genealogical development across the modes of production is the condition of possibility of the understanding of the process of history according to dialectical materialism, until that stage of development is reached in which life enters into a profound contradiction with its material reproduction. Understanding each historical moment in the light of the contradictions among the material productive forces, the existing relations of production, and their legal forms in relation not to matter but to life, that is, the subject, gives a new meaning to the understanding of history as process and not as progress. All of this raises moral issues and the unfathomable problem of the relations among the bourgeois mode of production, life, the appropriation of labor, and the logic of capital.

Keywords: materialism, dialectics, religion, capital, commodities.

\section{Vida e historia en el pensamiento de Marx}

Mientras Aristóteles cimentó los fundamentos de la cultura occidental, Marx nos enseñó los caminos que la historia debe recorrer. A pesar de las infinitas disensiones posibles entre ambos pensadores, también es posible encontrar puntos de convergencia para reinterpretar el mundo. Uno de estos puntos es la teoría aristotélica del hilemorfismo, según la cual todo cuerpo está compuesto por forma y materia. Admitamos como un 
principio autoevidente el mundo material, pero deduzcamos inevitablemente que al conjunto de la materia corresponde también una forma definitiva: la vida.

En abstracto, al conjunto de la materia corresponde una forma en concreto que es la vida: en Aristóteles la materia es ante todo potencia determinada por su receptividad de forma, siendo el movimiento un cambio en la forma de la sustancia. Esto quiere decir específicamente que el ser humano constituye la realidad conforme a su intención, transforma a todas las cosas en función de la satisfacción de sus necesidades. En el inacabable sistema hegeliano ya se planteaba la autonomía del hombre por su posición en el mundo: la propia de su actividad libre y racional. La realidad es y debe ser racionalizada, lo que arroja como resultado necesario una historia racional. Estos preceptos, propios de las revoluciones burguesas y apoyados en el floreciente proceso del capitalismo industrial, parecían capaces de garantizar los medios para satisfacer todas las necesidades humanas. Tal es el gran relato capitalista. Sin embargo, no es de este idealismo de lo que se trata. En Hegel podemos, más que superar, complementar la lógica formal con la lógica dialéctica, lo que permite reconocer el desarrollo del ser como un proceso que evoluciona a partir de contradicciones determinantes del contenido y el movimiento de toda la realidad. Y a pesar de esto, la dialéctica sería insuficiente.

De lo que se trata es de superar ese idealismo como lo hizo en su tiempo Marx, comprendiendo el proceso dialéctico, pero a partir de la vida como fundamento y no ya de la materia pues de ser esta lo principal, se deduce apodícticamente la revolución sin ninguna consideración acerca de la realidad. Por el contrario, con la vida como punto de partida y como forma de la materia podemos tener condiciones de análisis más complejas, amplias y concretas, sin eliminar las particularidades, sino enriqueciendo la interpretación del mundo. No se trata de copiar la dialéctica histórica adaptándola a circunstancias reales, o mejor, de suplantar la idea por la materia para inferir objetivamente las condiciones de posibilidad de la revolución, sino de fundar una auténtica filosofía materialista.

\section{Dialéctica entre lo material y lo formal}

Dice Marx en el "Prólogo a la contribución de la filosofía del derecho de Hegel”: "El fundamento de la crítica de la religión es: el hombre hace la religión y no ya, la religión hace al hombre” (1975a, p. 11). Mutatis mutandis, es posible preguntar: ¿Por qué debemos admitir que la materia hace al hombre y no ya este a aquella? El punto es el siguiente: los pensadores del materialismo histórico terminaron inconscientemente reproduciendo 
preceptos de la ideología burguesa. Para un liberalismo acérrimo está más que justificada la primacía de la materia. Esta, como propiedad, convierte la actividad del sujeto de trabajo en fuente de valores de uso, y por tanto, de riqueza. Ahora bien, el sujeto que no tiene más propiedad que su fuerza de trabajo necesariamente se transforma en objeto de dominación de quienes se han apropiado de las condiciones materiales de trabajo y queda sin bases la proposición de que la riqueza es solamente producto de este.

Por otro lado, la supuesta primacía de la materia en el análisis materialista provee objetividad en la investigación. Luego, los resultados exceden la voluntad del ser humano y el postulado revolucionario se vuelve una cuestión inevitable de la historia. Así, esta debe avanzar sin duda hacia la revolución, y la presente exposición no puede confundirse con una diatriba en contra de la materia ${ }^{1}$. Se trata de ampliar el análisis del materialismo histórico reduciendo el espectro del error. Por ejemplo, en términos reales la materia como principio es la base de la teoría sociológica del marxismo: una infraestructura que es base real de la sociedad y determina la superestructura jurídica y política. Pero ahí nuevamente encontramos más semejanzas con los preceptos de la burguesía liberal que con el pensamiento de Marx. Lo cierto es que esta visión incluso excusa el libre mercado porque, al igual que la perspectiva burguesa, pone el énfasis en la economía sin considerar las formas jurídicas, que determinan las relaciones de propiedad, o la forma política, que acredita a la economía. Evidentemente, y tal como Marx (1953) lo anuncia en los Grundrisse, las relaciones económicas pueden desarrollarse dentro de la sociedad burguesa determinadas, por ejemplo, por la guerra, entre otras causas.

De esto colegimos, como insiste Dussel, que los sistemas sociales son determinaciones determinantes determinadas porque es notable la correlación entre los componentes de un Estado. Por ejemplo: la primera revolución inglesa representaba todos los intereses de la burguesía y tenía como centro de disputa al parlamento inglés y al soberano. Las disputas entre el poder parlamentario y el poder absoluto devinieron en la conformación de la república, cuyo mayor impacto fue la declaración de las cartas de navegación. Estas leyes definieron el provenir de Inglaterra hasta convertirla en la potencia más importante del mundo en su momento, expandiendo su dominio desde lo naval hasta la comercial e impulsando el desarrollo industrial, todo mediado por una ideología. Este hecho histórico demuestra la complejidad de las relaciones existentes: la manera en

1 En especial considerando que toda revolución precisa de una base material. 
que los intereses económicos de la burguesía influenciaron el dominio político, cómo la política definió la legislación, la forma en que estas leyes determinaron el comercio y la manera en que el conjunto de estas determinaciones determinó a su vez la composición del Estado inglés. En resumen, la consideración de la base material de la sociedad no es el origen de una superestructura política e ideológica, sino que la economía es determinación en tanto determinada. Es decir, hay que considerar a la sociedad como una unidad y no como una jerarquía de estructuras.

Dicho de otro modo, en un sentido estricto la concepción materialista de sociedad es lo que más agrada a la ideología liberal pues implica la separación entre la economía, la moral, la política y el derecho, y más aún, la primacía de la economía, que cae como anillo al dedo de los intereses burgueses.

Es por esto que debemos adoptar un principio formal como base real de la sociedad, a saber, la vida, y considerar a la economía, la moral, la política y el derecho en su conjunto. Volvamos a la crítica del derecho de Hegel: "Quien dice el hombre, dice el mundo del hombre, Estado, sociedad” (Marx, 1975a, p. 11). Y si partíamos de la crítica de la religión, que es una conciencia subvertida del mundo, seguiremos con la crítica de la conciencia real de este, la economía, adaptándola al principio formal que hemos establecido. En esto consiste, entonces, nuestra dialéctica entre la forma y el contenido como dos estados de la conciencia en el sentido hegeliano, si se prefiere. De modo que la forma es la vida, y en particular la vida humana inmediata, y el contenido es todo el sistema social, o en este sentido, la relación entre la vida y su producción material.

\section{Contradicción entre capital y vida}

Dice Marx (2003) que la historia la hace el hombre bajo el legado del pasado. Esto es, el hombre hace la historia, pero no la hace conforme a su albedrío. Veamos entonces cómo fundar una nueva concepción materialista a partir de la vida, volviendo a la infinita fuente del joven Marx (Marx y Engels, 1975). Con la vida humana inmediata como principio, el primer hecho histórico es la producción material de la vida misma, es decir, de los medios indispensables para la satisfacción de las necesidades humanas - ejecución del principio de conservación-. Ya vimos cómo el sujeto deseante constituye todas las cosas como posibles fuentes de satisfacción de sus necesidades. $\mathrm{O}$ sea, mide si algo es útil o inútil. Por otro lado está el sujeto productor, que tiene la capacidad de constituir a la cosa como un producto posible. 
Tenemos en la producción material de la vida la primer relación social, la del sujeto deseante y el sujeto productor. Y en este último, la primera relación práctica: entre el sujeto y la naturaleza. El conjunto de estas dos relaciones compone la economía.

Sin duda, no se nos puede acusar de idealistas, en el sentido de Fedor Konstantinov, por enfocar nuestros esfuerzos en el ser humano como punto de partida porque nuestro fundamento no es el ego cogito sino el ego laboro. En efecto, la relación práctico-productiva esencial, la economía, se fundamenta en la relación que constituye a la naturaleza como materia de trabajo para la producción. No hemos tomado deferencia por ningún punto, como tampoco hemos despreciado a ninguno. Simplemente, como lo venimos anunciando, hemos dado al mundo material la formalidad sustancial de la vida, y en la economía tenemos la vida inmediata como principio formal.

La utilidad, esto es, el valor de uso, es fruto de la transformación en producto de una cosa en estado de composición natural. Y el producto dispuesto para el intercambio es la mercancía, a la que pueden atribuirse en este punto tres determinaciones: su valor de uso, su valor de cambio y su valor constituido por la relación técnica y el trabajo vivo. Ahora bien, en diferentes proporciones estas determinaciones constituyen la riqueza de las sociedades capitalistas. Dichas categorías económicas las podemos encontrar en la abstracción de la producción y la circulación. Sin embargo, es menester verificar en qué se diferencia el régimen capitalista de cualquier otro modo de producción.

En el sistema capitalista la producción de mercancías obedece al principio de rentabilidad. Aquellas ya no son producidas por su valor de utilidad intrínseco, sino con el valor de cambio como propósito. Es decir, para el sujeto productor el valor de uso de la mercancía es su valor de cambio, su quid pro quo. Tenemos aquí, desde la acumulación originaria de capital, que las relaciones prácticas productivas están mediadas por el proceso contractual del burgués y el obrero. Las relaciones sociales de producción se dan entre el propietario de los medios de producción y el propietario de la fuerza de trabajo, que como ya vimos no dispone de ninguna otra propiedad. Recordemos a la vez que la esencia subjetiva de la propiedad es el trabajo y que el trabajo objetivado compone la riqueza. Estas categorías son claves para comprender el crimen del capitalismo.

En la producción los obreros venden su fuerza de trabajo como una mercancía a cambio de dinero. El valor de cambio de una mercancía expresado en dinero es su precio y este expresa la proporción de valor de cambio de la fuerza de trabajo. O sea, el salario es el 
precio de la fuerza de trabajo. Aquí seguimos teniendo los dos principios: el formal, representado en el obrero vendiendo su fuerza de trabajo para poder vivir, para reproducir su vida material, y el material, un contenido objetivo que es el capital y el trabajo objetivo. Pero no debemos soslayar las posiciones de las dos voluntades: el obrero, que hace parte de la relación social de producción por necesidad, para poder reproducir su vida, y el burgués, que está inmerso en la acumulación de riqueza, de trabajo objetivado, embebido en la tasa de ganancia y la ley de rentabilidad.

Hemos establecido que el trabajo objetivado es la riqueza y que en la producción es el obrero quien emplea su fuerza de trabajo. Entonces, ¿por qué el creador de la riqueza carece de ella? La teoría del valor-trabajo sugiere que el valor de un producto está determinado por la cantidad de trabajo socialmente requerido para su producción. Pero, como Marx demuestra, el obrero recibe un salario a cambio de su fuerza de trabajo y no por su trabajo, lo que significa que el precio de aquella es apenas lo necesario para su reposición y la subsistencia del trabajador. Sin embargo, en la reproducción de su fuerza de trabajo el obrero gasta más tiempo del necesario para reproducir su vida creando un plusvalor que no le es retribuido. La plusvalía es entonces el excedente de trabajo del obrero, que restado a los costos de producción constituye la tasa de ganancia del capitalista. Ahora bien, esta ganancia está condicionada por un límite absoluto. Es decir, el plusvalor absoluto tiene un límite absoluto que es la vida del obrero pues su salario no debe ser menor que el costo de la reproducción de su vida. Y aunque el capitalista racionalice el proceso productivo para maximizar la ganancia a través de la división y especialización del trabajo o la aceleración de la producción, siempre va a encontrar el mismo límite absoluto.

¿Cómo aumentar la tasa de ganancia superando el límite absoluto? Disminuyendo la proporción del salario en el valor del producto, es decir, disminuyendo el tiempo necesario para su producción, lo que aumenta el plusvalor ya que la proporción del valor disminuye en el valor objetivado del producto. Esto ocurre a su vez en el marco de la ley de competencia. El capitalista necesita ser competente para aumentar sus beneficios, y lo logra a partir de la máquina. La competencia no crea valor, lo distribuye. Y así quien desarrolle una mejor base técnica, es decir, quien posea mejores instrumentos de producción, mejor base material de trabajo, mayor despliegue y alcance tecnológico en el mercado, determina el precio medio. Y quien no ha desarrollado las mismas capacidades técnicas ni tecnológicas transfiere valor al más competente, lo cual hace que se tienda a un principio de monopolio en el mercado. 
Cuando el salario tiene menos proporción en el producto, a causa de la disminución del tiempo de trabajo requerido para la producción de aquel, aparece el plusvalor relativo, lo cual eleva significativamente la tasa de ganancia del capitalista al reducir el valor de producto mediante el aumento constante de la producción. Pero esto tiene una consecuencia gravísima para el capitalismo: este movimiento objetivo de comenzar a preferir la inversión en capital constante en vez de hacerlo en capital variable - es decir, de desplazar el trabajo humano por la máquina - es la tragedia del capital, el arma que la burguesía forjó para su propia muerte: la ley de la tendencia decreciente de la tasa de ganancia.

Esta tendencia de reemplazar fuerza de trabajo por capital constante en el incremento de la ganancia para crear plusvalor es una contradicción, ya que el factor constante solamente aumenta a medida que el número de obreros se reduce, lo cual produce que se deprima la tasa de ganancia.

Ahora bien, son dos las contradicciones. La primera es que es imposible crear plusvalor sin trabajo vivo mientras la tendencia decreciente de la tasa de ganancia deviene en toda clase de fenómenos sociales, como el "ejército de reserva del capitalismo" o la ocupación de la población en otros sectores para su supervivencia. La segunda es, como ya lo habíamos anunciado, que la reducción del trabajo a una propiedad deviene en que quien no tenga más propiedad que su fuerza de trabajo es necesariamente esclavo de otros hombres. En efecto, el obrero depende de la voluntad de quien se ha adueñado de sus condiciones materiales para existir, y por consiguiente se ve obligado a vivir bajo su permiso. En suma, la apropiación del trabajo del otro es un atentado en contra de la reproducción de la vida del obrero, con lo cual es evidente que el capital niega el principio de conservación que hemos establecido antes. Bajo esta contradicción, sumada al contenido objetivo ${ }^{2}$, se justifica una revolución social.

\section{Elementos a considerar}

Si tenemos presentes los términos objetivos y materiales que acabamos de estudiar, bajo la mirada de la teoría sociológica del marxismo tradicional a toda luz la revolución se hace un hecho irresistible. Pero, como hemos venido insistiendo, es necesario ampliar el análisis y verificar por qué, más allá de la correlación de fuerzas entre elementos revolucionarios y reaccionarios, no ha sido posible una revolución socialista. Como veníamos

2 Al llegar a una determinada fase de desarrollo, las fuerzas productivas materiales entran en contradicción con las relaciones de producción existentes. 
anunciando, es sencillo deducir la necesidad de la revolución a partir del análisis de la materia, y más cuando se tienen fundamentos como la ley de tendencia decreciente de la tasa de ganancia. A pesar de ello, aquella proposición apodíctica se hace contingente, así que debemos expandir el espectro de nuestra investigación. Para eso hemos porfiado en sugerir un principio formal, la vida inmediata, y un contenido que no es solamente la base material de la sociedad, sino la sociedad en su conjunto. Con estos elementos se demuestra la infinita complejidad que supone la transformación de una estructura social pues, admitiendo que el cambio de la base material acelera el proceso, no es suficiente para augurar una revolución.

Primero debemos considerar que

[...] ninguna formación social desaparece antes de que se desarrollen todas las fuerzas productivas que caben dentro de ella, y jamás aparecen nuevas y más altas relaciones de producción antes de que las condiciones materiales para su existencia hayan madurado en el seno de la propia sociedad antigua. (Marx, 1975b, p. 8)

Recordemos que aunque el hombre hace la historia lo hace dependiendo de condiciones materiales concretas y que además el ser humano únicamente se propone objetivos realizables.

Segundo, debemos admitir que en el entramado de relaciones sociales muchas esferas diferentes a la economía influyen en las condiciones de posibilidad de una revolución, y que estas determinan al tiempo que son determinadas, lo cual hace que el planteamiento esté revestido de un sinnúmero de problemas que se extiende hasta la más mínima particularidad.

Para revisar la anterior proposición voy a limitar este estudio a un caso particular, sin querer insinuar que se trata de un caso excepcional. Al contrario, no se trata de suponer que es un hecho aislado, sino todo lo opuesto, afirmar que forma parte de un conjunto más amplio de determinaciones todas las cuales operan de la misma manera y no hay espacio para la excepción.

El tema que vamos a revisar para analizar más a fondo una perspectiva distinta de la concepción material de la realidad es la falacia naturalista, el problema del ser y el deber 
ser. Los más avezados filósofos, como Hume, han planteado dicho problema advirtiendo una cantidad de dificultades al tratar de usar el ser deontológicamente. Según Moore el inconveniente radica en pretender inferir lo "bueno" de lo natural, así que a partir de esta cuestión moral vamos a evidenciar la articulación real entre una fase de desarrollo de un modo de producción y una condición revolucionaria específica.

En la época del ascenso de la burguesía, con los latentes conflictos de intereses entre el antiguo régimen y las aspiraciones capitalistas crecientes, surge un razonamiento moral, la falacia naturalista, que se presenta del siguiente modo: el hombre tiene sentimientos, ya sean positivos o negativos; el mayor sentimiento negativo del hombre es la avaricia, es decir, la pretensión universal de un dominio absoluto; para regular ese sentimiento de dominio total es necesario limitar su alcance otorgando propiedad a un grupo específico de personas. Como podemos observar es un razonamiento de carácter moral que presenta pretensiones de limitar el poder político absolutista del antiguo régimen a través de la propiedad. Naturalmente, esta concepción moral estaba presente en la conciencia burguesa y significaba que su aplicación real configuraba determinadas formas jurídicas de la propiedad. Esto es, las relaciones de propiedad expresadas en el derecho estaban ligadas a una moral definida por la contradicción entre las fuerzas materiales de la burguesía ascendente y las relaciones de producción absolutistas. En otras palabras, la moral determinada por la economía determina el derecho, y el derecho determina la política, que a su vez determina a la economía. Determinaciones determinantes determinadas.

Así opera la moral revolucionaria, pero cuando una argumentación naturalista justifica un poder que se reforma virando hacia la reacción, como la falacia naturalista del capitalismo, el razonamiento es el siguiente: es natural la desigualdad en el hombre; siempre han existido ricos y pobres, dominados y dominadores porque la naturaleza humana es dominadora, siempre apetece más. Se advierte la enorme dificultad que supone este discurrir, más aún cuando el convencimiento general acerca de esto es profuso y contundente. El capitalista triunfa en su propósito y se perpetúa la desigualdad económica, la apropiación del trabajo del otro, pues, aunque las condiciones materiales dictan la necesidad de una revolución social, en la conciencia intersubjetiva se ve al capitalismo como algo natural y no como un momento histórico. Con lo cual volvemos al mismo punto: no basta con modificar la base material, es necesario también transformar la conciencia, la moral, la legislación, la política, la educación y el derecho. Es decir, remover los fundamentos en conjunto de la antigua sociedad antes de proceder a intentar 
cambiarla porque de nada sirve avanzar en la transformación de su base real si en la conciencia la misma clase obrera, por ejemplo, tiene preceptos burgueses, reaccionarios $\mathrm{o}$ antediluvianos.

El mismo resultado tendríamos si se prefiere analizar el derecho o la política en lugar de la moral. En este caso aplicamos la última porque significa la relación social práctica —o sea, hombre-hombre- más simple pero más compleja de enfrentar. Es indudable que en la política la relación social es bastante compleja en relación con la moral, pero más sencilla de confrontar por ser más evidente sus contradicciones.

\section{Situación concreta}

¿Qué podemos concluir de toda esta exposición para Colombia? Lo cierto es que aplicar el análisis a nuestra situación enfrenta disímiles barreras y multiplica las dificultades; las categorías se hacen más complejas y los conceptos, más confusos. Las condiciones materiales del país y su anormal fase de desarrollo son una contradicción en sí misma. Colombia es una nación con relaciones de mercado en el capital global, pero ha sido incapaz de alcanzar un estado propiamente capitalista. En este sentido, en palabras de Marx, "somos contemporáneos filosóficos del presente sin ser contemporáneos históricos” (Marx, 1975a, p. 16). Si cambiamos filosofía por ideología nuestra situación actual es risiblemente similar a la de Alemania en 1844, pero trágicamente ambigua. Me refiero precisamente a lo que Marx denunciaba en su crítica a la filosofía del derecho de Hegel: "La filosofía alemana del Derecho y del Estado es la única historia alemana que está a la par con el oficial tiempo moderno" (Marx, 1975a, p. 17). De la misma manera, Colombia está dotada de toda una estructura republicana, con división de poderes y dinámicas económicas de libre mercado, pero con un aparato productivo insignificante, relaciones sociales de producción semifeudales y una condición de víctima del capital extranjero.

Colombia ha atravesado etapas convulsas y una historia radical. El cambio de orden a nivel internacional no ha afectado sus estructuras, no ha podido superar ninguna fase histórica y parece estar petrificada en un antiguo régimen. Así como Marx demolía el pensamiento ajado de la Alemania de 1843 en comparación con la Francia de 1789, en Colombia no estaríamos cerca de comparar el presente ni con el periodo de Luis XIV, por más esfuerzos de transformación que intenten ciertos sectores políticos. Sin embargo, nuestra situación es más penosa todavía porque la nuestra es la historia de la lisonja, la credulidad y la indigencia, de la postración y la humillación. ¿Acaso en 1903 no ocurrió 
el primer clarividente ejemplo de nuestra ingenuidad y nuestra postración, y acaso este estado de miseria no se ha perpetuado hasta nuestros días más próximos con la maltrecha entrada del país en la Ocde?

Después de la Guerra de los Mil Días y hasta la década de los treinta la economía colombiana logró consolidarse medianamente en dos sectores: el cafetero y el industrial (sobre todo manufacturero), siendo estos la piedra de toque de las conciliaciones entre los legisladores de la política económica hasta los noventa. Sin embargo, este desarrollo tuvo toda clase de contrariedades. En los años cincuenta las fuerzas productivas resultaron dañadas por la relación metrópoli-colonia: Colombia comenzaba ya a perfilar todo su potencial subordinado renunciando a sus productos agrícolas más esenciales hasta limitarse exageradamente a productos tropicales. En los noventa termina de definirse la división mundial del trabajo y América, y en particular Colombia, que atravesaron desde la doctrina Monroe hasta los conflictos geopolíticos del siglo xxi haciendo el papel de zona de influencia de Estados Unidos, consolidan su dependencia con respecto al vecino del norte. ¿Qué significó todo esto en términos concretos? El origen de la tragedia: la apertura económica. Con la nueva época de globalización y las nuevas medidas económicas el país terminó duplicando tan solo en seis años, entre 1992 y 1998, su deuda externa, que llegó a más de diecisiete millones de dólares en ese momento y ha alcanzado hoy día más de trescientos billones de pesos.

La ideología que comenzó a predominar desde los noventa — asociada a las políticas del nuevo liberalismo que se aplican en Colombia por recomendaciones del FMI y el BID, entre otros - de reducir el agro y la industria frente a la competencia extranjera trajo terribles consecuencias para el país. Por ley de competencia Colombia pasó a transferir valores en el mercado mundial, igual que en el proceso del capital que vimos anteriormente. Y por su deficiente base técnica y tecnológica producía a precios bastante altos y con costos de producción superiores a los beneficios percibidos. Todo eso devino inevitablemente en una afectación en la balanza comercial: incrementó enormemente el valor de las importaciones por encima de las exportaciones, que siguen siendo los productos de siempre - café, banano, flores, petróleo, carbón, etc. - y no tienen un impacto importante en la base real de la economía colombiana. A causa de esto las importaciones del sector agropecuario fueron aproximadamente de 7 millones de toneladas con respecto a las $700000 \mathrm{t}$ del registro anterior, lo que sumado a todas las vicisitudes del campo colombiano y a la crisis del café le terminó entregando al neoliberalismo un nuevo mercado y miles de nuevos miserables colombianos para explotar. De la misma 
forma, a este fenómeno se sumó el sector manufacturero, que contó con una suerte incluso peor, sufriendo entre 1993 y 1998 una disminución de 5.9 \% en su contribución al PIB, lo que significó el lento suicidio de un imprescindible sector industrial colombiano.

En consecuencia, viéndose afectada la producción urbana y rural, el conjunto de la economía colombiana sufrió la peor crisis de su historia, afectando el empleo, los salarios, las empresas y las utilidades. Es decir, Colombia pasó de tener retoños de capitalismo al atraso semifeudal. Y la crisis se sigue perpetuando. Los tratados de libre comercio juegan en detrimento del bienestar de la nación porque se erigen sobre la ficción de la igualdad, cuando hemos visto la culpable incompetencia de Colombia fruto de un exiguo aparato productivo. Esto a su vez es consecuencia de la fervorosa aplicación de las medidas del neoliberalismo, que han convertido al país en dependiente de todo lo que ya no puede producir. Como en el proceso del capital que vimos anteriormente, se vive bajo el permiso de otro y profundizando la desigualdad social en el seno del propio territorio, lo que ha convertido a Colombia en uno de los países con peor distribución de la riqueza.

Entonces, ¿qué sostiene a la economía colombiana? La inversión de capital extranjero, incentivada por la confianza inversionista, la eliminación del impuesto sobre las remesas y un sinfín de arbitrariedades que se justifican porque mantiene la lánguida base material del país.

Y si la contradicción ética del capital es tomar una persona como instrumento del aumento de la tasa de ganancia, la contradicción imperio-nación a la que estamos sujetos consiste en tomar a todo un país en función de la rentabilidad. Es decir, ya no nos jugamos la vida del obrero, como en el capitalismo. En el imperialismo la vida abstracta de una nación se pone en riesgo y por las mismas razones: la apropiación de su trabajo objetivado y la dependencia de los designios del dominador para poder reproducir la vida. Las consecuencias son más que obvias, basta con fijar la mirada en la periferia de las capitales del país para reconocer la extracción de valor objetivada en el territorio.

Y si realmente la contradicción es tan grande y penosa, si las condiciones materiales están en conflicto con las relaciones sociales, ¿por qué no se ve en el horizonte la posibilidad de una revolución social, aunque sea una burguesa, que permita el desarrollo del capitalismo? La razón es que no basta la precariedad de la base material como motivo. Es necesario eliminar la felicidad ilusoria, "hacer más oprimente la opresión real añadiéndole conciencia de opresión” (Marx, 1975a, p. 14) y destruir los falsos ídolos que encubren 
y ocultan estas contradicciones. El ejercicio está, pues, en considerar a la sociedad en su conjunto y ofrecer una alternativa, enseñando cada punto coyuntural que ha sumido a la nación en el atraso y la miseria. Se trata de la tarea pedagógica más importante de la historia de Colombia. En definitiva hemos carecido de una crítica como la que proponía el joven Marx: no una pasión del cerebro, sino el cerebro de una pasión.

\section{Referencias}

Marx, K. (1953). Grundrisse: elementos fundamentales para la crítica de la economía política. Madrid: Siglo XXI.

Marx, K. (1975a [1844]). Prólogo de la contribución a la filosofía del derecho de Hegel. En K. Marx y F. Engels, Obras escogidas (pp. 11-26). Bogotá: Túpac Amaru.

Marx, K. (1975b [1859]). Prólogo de la contribución a la crítica de la economía política. K. Marx y F. Engels, Obras escogidas (pp. 168-173). Bogotá: Túpac Amaru.

Marx, K. (2003 [1852]). 18 Brumario de Luis Bonaparte. Madrid: Fundación Federico Engels.

Marx, K. y Engels, F. (1975 [1932]). La ideología alemana. En Obras escogidas (pp. 27-92). Bogotá: Túpac Amaru. 